\title{
NCPDP Dose Unit of Measure Terminology
}

National Cancer Institute

\section{Source}

National Cancer Institute. NCPDP Dose Unit of Measure Terminology. NCI Thesaurus. Code $C 121847$.

A set of terminology for NCPDP that contains concepts of the intended dose unit of measure (e.g. tablet, drop, puff, $\mathrm{mL}$, lozenge) as presented in the medication directions for use (sig). The purpose of this subset is to state dosing quantities, therefore the concepts contained within may not be the same, or appropriate, units used for dispensing/billing. 